\title{
Simulations of RS Oph and the CSM in Type Ia Supernovae
}

\author{
Richard A. Booth ${ }^{1}$, Shazrene Mohamed ${ }^{2}$ and Philipp Podsiadlowski ${ }^{1}$ \\ ${ }^{1}$ Dept. of Astrophysics, University of Oxford, \\ Denys Wilkinson Building, Keble Road, Oxford, OX1 3RH, United Kingdom \\ email: richard.booth@astro.ox.ac.uk, podsi@astro.ox.ac.uk \\ ${ }^{2}$ South African Astronomical Observatory \\ Observatory Road, Observatory, 7925, South Africa \\ email: shazrene@saao.ac.za
}

\begin{abstract}
Smoothed particle hydrodynamics (SPH) models of the recurrent nova RS Ophiuchi are presented, along with simple models for circumstellar absorption lines. The evolution of the model sodium line is similar to the behaviour in some Type Ia SNe, e.g. SN2006X.
\end{abstract}

Keywords. hydrodynamics, binaries: symbiotic, circumstellar matter, stars: individual (RS Oph), stars: novae, cataclysmic variables, supernovae

\section{Introduction}

The explosion of a Chandrasekhar-mass white dwarf (WD) accreting from a companion is one popular model for Type Ia Supernovae (SNe Ia), but the nature of the companion is still being debated. The circumstellar material (CSM) constrains the mass accretion process and therefore provides potential insight on the nature of the companion. In the case of a red gaint (RG) companion, there is an equatorial wind and the possibility of recurrent novae, as is observed in the Galactic symbiotic binary RS Oph. SPH simulations are used to model the CSM in RS Oph and investigate the CSM seen in some SN Ia.

\section{Circumstellar model}

The interaction of the RG wind with the binary potential is modelled with the $3 \mathrm{D}$ SPH code GADGET-2 (Springel 2005), which has been modified as described in Mohamed (2010). The binary parameters are the same as in Mohamed et al. (2013). The RG and WD masses are 0.8 and $1.38 M_{\odot}$, and the binary separation is $1.48 \mathrm{AU}$. The wind massloss rate and velocity are $10^{-7} M_{\odot} \mathrm{yr}^{-1}$ and $20 \mathrm{~km} \mathrm{~s}^{-1}$, respectively. The binary potential focuses the wind into the equatorial plane and forms an Archimedian spiral shock.

Every 20 years a nova is ejected into the system. The nova shell is spherically symmetric with a mass of $2 \times 10^{-7} M_{\odot}$, and velocity of $4000 \mathrm{~km} \mathrm{~s}^{-1}$. The equatorial wind constrains the nova, which results in a bipolar structure. The evolution of the novae is followed for 80 years, including the ejection of three novae and their interaction with the previous novae. Fig. 1 (left) shows the system roughly 2 years after the 3rd nova. Components from all three novae can be seen. There is also a smooth equatorial component, which is material from the 1st nova that has been shock heated by the 2 nd nova.

\section{Circumstellar Sodium Absorption in SN Ia}

The CSM 20 years after the 3rd nova is used to model sodium absorption as seen in some SN Ia. The material is assumed to be fully ionized for the first 17 days after 

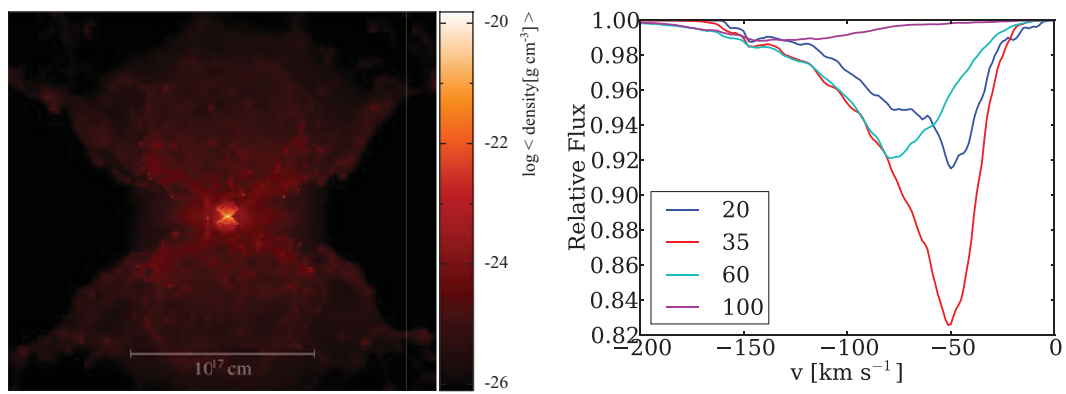

Figure 1. Left: Density Profile for a model of RS Oph 2 years after the 3rd nova. Right: Sodium absorption line evolution against a SN, for an observer aligned with the plane of the orbit. Recombination gives rise to the initial deepening of the line after the UV flux has decreased. The SN sweeping up the CSM and the decreasing covering factor of the densest regions causes the lines to weaken.

the explosion, after which it recombines on timescales of 10-15 days. This leads to an initial deepening of the line, until the supernova begins to sweep up the material, and the photosphere becomes larger than the equatorial waist. The line begins to weaken thereafter, with the lowest velocity components disappearing first. Fig. 1, (right) shows the evolution, which has features similar to those seen in SN2006X (Patat et al. 2007) and SN2007af (Simon et al. 2009).

Densities of $10^{5} \mathrm{~cm}^{-3}$ are required in the nova shell to reproduce the 10-15 day recombination time scale, however the simulations produce densities of a few $100 \mathrm{~cm}^{-3}$. The formation of optically thin micro-clumps may be able to explain the discrepancy, as may be necessary to explain the apparently super-Eddington mass loss rates from massive stars (Gräfener 2008). Small-scale clumping increases the recombination rate (which depends on the square of the density), without increasing the mean density. An alternative explanation is shell thickness. The simulations currently produce nova shell thickness $\Delta r / r \approx 1$, which maybe much thicker than the shells produced in nature. For example, Patat et al. (2007) suggest a thickness $\Delta r / r \approx 0.01$ for the shells in SN2006X. An investigation into nova shells is now being conducted.

\section{Conclusions}

The detection of CSM in some SN Ia provides a way to test the progenitor channels of at least some of the supernovae. Preliminary models of sodium absorption lines from 3D SPH models of the CSM show some of the features seen in SN2006X. A systematic investigation into the line profiles is being conducted to compare them to a broader range of SNe Ia.

\section{References}

Gräfener G., 2008, in: W.-R. Hamann, A. Feldmeier, L. M Oskinova (eds.), Clumping in Hot-Star Winds, p. 103

Mohamed, S., 2010, Ph.D. thesis, Univ. Oxford

Mohamed, S., Booth, R., \& Podsiadlowski, P., 2013, in: R. Di Stefano, M. Orio, \& M. Moe (eds.), Binary Paths to Type Ia Supernovae Explosions, Proc. IAU Symposium No. 281, (Cambridge), p. 195

Patat, F., Chandra, P., Chevalier, R., Justham, S., et al. 2007, Science, 317, 924

Simon, J. D., Gal-Yam, A., Gnat, O., et al. 2009, ApJ, 702, 1157

Springel, V. 2005, MNRAS, 37, 239 